\title{
Search for Lepton-Flavor and Lepton-Number Violation in the Decay $\boldsymbol{\tau}^{-} \rightarrow \ell^{\mp} h^{ \pm} h^{\prime-}$
}

B. Aubert, ${ }^{1}$ R. Barate, ${ }^{1}$ D. Boutigny, ${ }^{1}$ F. Couderc,${ }^{1}$ Y. Karyotakis,,${ }^{1}$ J. P. Lees, ${ }^{1}$ V. Poireau, ${ }^{1}$ V. Tisserand, ${ }^{1}$ A. Zghiche, ${ }^{1}$ E. Grauges, ${ }^{2}$ A. Palano, ${ }^{3}$ M. Pappagallo, ${ }^{3}$ A. Pompili, ${ }^{3}$ J. C. Chen, ${ }^{4}$ N. D. Qi,${ }^{4}$ G. Rong, ${ }^{4}$ P. Wang,${ }^{4}$ Y. S. Zhu, ${ }^{4}$ G. Eigen, ${ }^{5}$ I. Ofte, ${ }^{5}$ B. Stugu, ${ }^{5}$ G. S. Abrams, ${ }^{6}$ M. Battaglia, ${ }^{6}$ A. B. Breon, ${ }^{6}$ D. N. Brown, ${ }^{6}$ J. Button-Shafer, ${ }^{6}$ R. N. Cahn, ${ }^{6}$ E. Charles, ${ }^{6}$ C. T. Day, ${ }^{6}$ M. S. Gill, ${ }^{6}$ A. V. Gritsan, ${ }^{6}$ Y. Groysman, ${ }^{6}$ R. G. Jacobsen, ${ }^{6}$ R. W. Kadel, ${ }^{6}$ J. Kadyk, ${ }^{6}$ L. T. Kerth, ${ }^{6}$ Yu. G. Kolomensky, ${ }^{6}$ G. Kukartsev, ${ }^{6}$ G. Lynch, ${ }^{6}$ L. M. Mir, ${ }^{6}$ P. J. Oddone, ${ }^{6}$ T. J. Orimoto, ${ }^{6}$ M. Pripstein, ${ }^{6}$ N. A. Roe, ${ }^{6}$ M. T. Ronan, ${ }^{6}$ W. A. Wenzel, ${ }^{6}$ M. Barrett, ${ }^{7}$ K. E. Ford, ${ }^{7}$ T. J. Harrison, ${ }^{7}$ A. J. Hart, ${ }^{7}$ C. M. Hawkes, ${ }^{7}$ S. E. Morgan, ${ }^{7}$ A. T. Watson, ${ }^{7}$ M. Fritsch, ${ }^{8}$ K. Goetzen, ${ }^{8}$ T. Held, ${ }^{8}$ H. Koch, ${ }^{8}$ B. Lewandowski, ${ }^{8}$ M. Pelizaeus, ${ }^{8}$ K. Peters, ${ }^{8}$ T. Schroeder, ${ }^{8}$ M. Steinke, ${ }^{8}$ J. T. Boyd, ${ }^{9}$ J. P. Burke, ${ }^{9}$ N. Chevalier, ${ }^{9}$ W. N. Cottingham, ${ }^{9}$ M. P. Kelly, ${ }^{9}$ T. Cuhadar-Donszelmann, ${ }^{10}$ B. G. Fulsom, ${ }^{10}$ C. Hearty, ${ }^{10}$ N. S. Knecht,,${ }^{10}$ T. S. Mattison, ${ }^{10}$ J. A. McKenna,${ }^{10}$ A. Khan, ${ }^{11}$ P. Kyberd,${ }^{11}$ M. Saleem, ${ }^{11}$ L. Teodorescu, ${ }^{11}$ A. E. Blinov, ${ }^{12}$ V. E. Blinov, ${ }^{12}$ A. D. Bukin, ${ }^{12}$ V. P. Druzhinin, ${ }^{12}$ V. B. Golubev, ${ }^{12}$ E. A. Kravchenko, ${ }^{12}$ A. P. Onuchin, ${ }^{12}$ S. I. Serednyakov, ${ }^{12}$ Yu. I. Skovpen, ${ }^{12}$ E. P. Solodov, ${ }^{12}$ A. N. Yushkov, ${ }^{12}$ D. Best, ${ }^{13}$ M. Bondioli, ${ }^{13}$ M. Bruinsma, ${ }^{13}$ M. Chao, ${ }^{13}$ I. Eschrich, ${ }^{13}$ D. Kirkby, ${ }^{13}$ A. J. Lankford, ${ }^{13}$ M. Mandelkern, ${ }^{13}$ R. K. Mommsen, ${ }^{13}$ W. Roethel, ${ }^{13}$ D. P. Stoker, ${ }^{13}$ C. Buchanan, ${ }^{14}$ B. L. Hartfiel, ${ }^{14}$ A. J. R. Weinstein, ${ }^{14}$ S. D. Foulkes, ${ }^{15}$ J. W. Gary, ${ }^{15}$ O. Long, ${ }^{15}$ B. C. Shen ${ }^{15}$ K. Wang, ${ }^{15}$ L. Zhang, ${ }^{15}$ D. del Re,${ }^{16}$ H. K. Hadavand,${ }^{16}$ E. J. Hill, ${ }^{16}$ D. B. MacFarlane, ${ }^{16}$ H. P. Paar, ${ }^{16}$ S. Rahatlou, ${ }^{16}$ V. Sharma, ${ }^{16}$ J. W. Berryhill, ${ }^{17}$ C. Campagnari, ${ }^{17}$ A. Cunha, ${ }^{17}$ B. Dahmes, ${ }^{17}$ T. M. Hong, ${ }^{17}$ M. A. Mazur, ${ }^{17}$ J. D. Richman, ${ }^{17}$ W. Verkerke, ${ }^{17}$ T. W. Beck, ${ }^{18}$ A. M. Eisner, ${ }^{18}$ C. J. Flacco, ${ }^{18}$ C. A. Heusch,${ }^{18}$ J. Kroseberg, ${ }^{18}$

W. S. Lockman, ${ }^{18}$ G. Nesom, ${ }^{18}$ T. Schalk,${ }^{18}$ B. A. Schumm, ${ }^{18}$ A. Seiden, ${ }^{18}$ P. Spradlin, ${ }^{18}$ D. C. Williams,${ }^{18}$ M. G. Wilson, ${ }^{18}$ J. Albert, ${ }^{19}$ E. Chen, ${ }^{19}$ G. P. Dubois-Felsmann, ${ }^{19}$ A. Dvoretskii, ${ }^{19}$ D. G. Hitlin, ${ }^{19}$ I. Narsky, ${ }^{19}$ T. Piatenko, ${ }^{19}$ F. C. Porter, ${ }^{19}$ A. Ryd ${ }^{19}$ A. Samuel, ${ }^{19}$ R. Andreassen,${ }^{20}$ S. Jayatilleke, ${ }^{20}$ G. Mancinelli, ${ }^{20}$ B. T. Meadows,${ }^{20}$ M. D. Sokoloff, ${ }^{20}$ F. Blanc, ${ }^{21}$

P. Bloom, ${ }^{21}$ S. Chen,${ }^{21}$ W. T. Ford, ${ }^{21}$ U. Nauenberg, ${ }^{21}$ A. Olivas,${ }^{21}$ P. Rankin,${ }^{21}$ W. O. Ruddick,${ }^{21}$ J. G. Smith, ${ }^{21}$ K. A. Ulmer, ${ }^{21}$ S. R. Wagner, ${ }^{21}$ J. Zhang, ${ }^{21}$ A. Chen, ${ }^{22}$ E. A. Eckhart, ${ }^{22}$ A. Soffer, ${ }^{22}$ W. H. Toki, ${ }^{22}$ R. J. Wilson, ${ }^{22}$ Q. Zeng, ${ }^{22}$ D. Altenburg, ${ }^{23}$ E. Feltresi, ${ }^{23}$ A. Hauke ${ }^{23}$ B. Spaan, ${ }^{23}$ T. Brandt,${ }^{24}$ J. Brose,${ }^{24}$ M. Dickopp,${ }^{24}$ V. Klose,${ }^{24}$ H. M. Lacker, ${ }^{24}$ R. Nogowski, ${ }^{24}$ S. Otto, ${ }^{24}$ A. Petzold, ${ }^{24}$ G. Schott, ${ }^{24}$ J. Schubert, ${ }^{24}$ K. R. Schubert, ${ }^{24}$ R. Schwierz, ${ }^{24}$ J. E. Sundermann, ${ }^{24}$ D. Bernard, ${ }^{25}$ G. R. Bonneaud, ${ }^{25}$ P. Grenier, ${ }^{25}$ S. Schrenk, ${ }^{25}$ Ch. Thiebaux, ${ }^{25}$ G. Vasileiadis, ${ }^{25}$ M. Verderi, ${ }^{25}$ D. J. Bard, ${ }^{26}$ P. J. Clark,${ }^{26}$ W. Gradl,${ }^{26}$ F. Muheim, ${ }^{26}$ S. Playfer, ${ }^{26}$ Y. Xie, ${ }^{26}$ M. Andreotti, ${ }^{27}$ V. Azzolini, ${ }^{27}$ D. Bettoni, ${ }^{27}$ C. Bozzi, ${ }^{27}$ R. Calabrese, ${ }^{27}$ G. Cibinetto, ${ }^{27}$ E. Luppi,${ }^{27}$ M. Negrini, ${ }^{27}$ L. Piemontese,${ }^{27}$ F. Anulli, ${ }^{28}$ R. Baldini-Ferroli, ${ }^{28}$ A. Calcaterra, ${ }^{28}$ R. de Sangro, ${ }^{28}$ G. Finocchiaro, ${ }^{28}$ P. Patteri, ${ }^{28}$ I. M. Peruzzi, ${ }^{28, *}$ M. Piccolo, ${ }^{28}$ A. Zallo ${ }^{28}$ A. Buzzo ${ }^{29}$ R. Capra, ${ }^{29}$ R. Contri, ${ }^{29}$ M. Lo Vetere, ${ }^{29}$ M. Macri, ${ }^{29}$ M. R. Monge, ${ }^{29}$ S. Passaggio, ${ }^{29}$ C. Patrignani, ${ }^{29}$ E. Robutti, ${ }^{29}$ A. Santroni, ${ }^{29}$ S. Tosi, ${ }^{29}$ S. Bailey, ${ }^{30}$ G. Brandenburg, ${ }^{30}$ K. S. Chaisanguanthum, ${ }^{30}$ M. Morii, ${ }^{30}$ E. Won, ${ }^{30}$ J. Wu, ${ }^{30}$ R. S. Dubitzky, ${ }^{31}$ U. Langenegger, ${ }^{31}$ J. Marks, ${ }^{31}$ S. Schenk, ${ }^{31}$ U. Uwer, ${ }^{31}$ W. Bhimji, ${ }^{32}$ D. A. Bowerman, ${ }^{32}$ P. D. Dauncey, ${ }^{32}$ U. Egede, ${ }^{32}$ R. L. Flack, ${ }^{32}$ J. R. Gaillard,${ }^{32}$ G. W. Morton, ${ }^{32}$ J. A. Nash,${ }^{32}$ M. B. Nikolich, ${ }^{32}$ G. P. Taylor, ${ }^{32}$ W. P. Vazquez, ${ }^{32}$ M. J. Charles, ${ }^{33}$ W. F. Mader ${ }^{33}$ U. Mallik, ${ }^{33}$ A. K. Mohapatra, ${ }^{33}$ J. Cochran, ${ }^{34}$ H. B. Crawley,${ }^{34}$ V. Eyges,${ }^{34}$ W. T. Meyer, ${ }^{34}$ S. Prell, ${ }^{34}$ E. I. Rosenberg, ${ }^{34}$ A. E. Rubin, ${ }^{34}$ J. Yi,${ }^{34}$ N. Arnaud,${ }^{35}$ M. Davier,${ }^{35}$ X. Giroux,${ }^{35}$ G. Grosdidier, ${ }^{35}$ A. Höcker, ${ }^{35}$

F. Le Diberder, ${ }^{35}$ V. Lepeltier, ${ }^{35}$ A. M. Lutz, ${ }^{35}$ A. Oyanguren, ${ }^{35}$ T. C. Petersen, ${ }^{35}$ M. Pierini, ${ }^{35}$ S. Plaszczynski, ${ }^{35}$ S. Rodier, ${ }^{35}$ P. Roudeau, ${ }^{35}$ M. H. Schune, ${ }^{35}$ A. Stocchi, ${ }^{35}$ G. Wormser, ${ }^{35}$ C. H. Cheng, ${ }^{36}$ D. J. Lange, ${ }^{36}$ M. C. Simani, ${ }^{36}$ D. M. Wright, ${ }^{36}$ A. J. Bevan, ${ }^{37}$ C. A. Chavez, ${ }^{37}$ J. P. Coleman, ${ }^{37}$ I. J. Forster, ${ }^{37}$ J. R. Fry, ${ }^{37}$ E. Gabathuler, ${ }^{37}$ R. Gamet, ${ }^{37}$ K. A. George, ${ }^{37}$ D. E. Hutchcroft,${ }^{37}$ R. J. Parry, ${ }^{37}$ D. J. Payne, ${ }^{37}$ K. C. Schofield,${ }^{37}$ C. Touramanis, ${ }^{37}$ C. M. Cormack, ${ }^{38}$ F. Di Lodovico, ${ }^{38}$ R. Sacco,${ }^{38}$ C. L. Brown, ${ }^{39}$ G. Cowan,${ }^{39}$ H. U. Flaecher, ${ }^{39}$ M. G. Green, ${ }^{39}$ D. A. Hopkins, ${ }^{39}$ P. S. Jackson, ${ }^{39}$ T. R. McMahon, ${ }^{39}$ S. Ricciardi, ${ }^{39}$ F. Salvatore, ${ }^{39}$ D. Brown, ${ }^{40}$ C. L. Davis,${ }^{40}$ J. Allison, ${ }^{41}$ N. R. Barlow, ${ }^{41}$ R. J. Barlow, ${ }^{41}$ M. C. Hodgkinson, ${ }^{41}$ G. D. Lafferty, ${ }^{41}$ M. T. Naisbit, ${ }^{41}$ J. C. Williams, ${ }^{41}$ C. Chen ${ }^{42}$ A. Farbin, ${ }^{42}$ W. D. Hulsbergen ${ }^{42}$ A. Jawahery, ${ }^{42}$ D. Kovalskyi ${ }^{42}$ C. K. Lae, ${ }^{42}$ V. Lillard, ${ }^{42}$ D. A. Roberts, ${ }^{42}$ G. Simi,${ }^{42}$ G. Blaylock, ${ }^{43}$ C. Dallapiccola, ${ }^{43}$ S. S. Hertzbach, ${ }^{43}$ R. Kofler, ${ }^{43}$ V. B. Koptchev, ${ }^{43}$ X. Li ${ }^{43}$ T. B. Moore ${ }^{43}$ S. Saremi,${ }^{43}$ H. Staengle, ${ }^{43}$ S. Willocq, ${ }^{43}$ R. Cowan, ${ }^{44}$ K. Koeneke, ${ }^{44}$ G. Sciolla,${ }^{44}$ S. J. Sekula, ${ }^{44}$ M. Spitznagel, ${ }^{44}$ F. Taylor, ${ }^{44}$ R. K. Yamamoto, ${ }^{44}$ H. Kim, ${ }^{45}$ P. M. Patel, ${ }^{45}$ S. H. Robertson, ${ }^{45}$ A. Lazzaro, ${ }^{46}$ V. Lombardo, ${ }^{46}$ F. Palombo, ${ }^{46}$ J. M. Bauer, ${ }^{47}$ L. Cremaldi, ${ }^{47}$ V. Eschenburg, ${ }^{47}$ R. Godang, ${ }^{47}$ R. Kroeger, ${ }^{47}$ J. Reidy, ${ }^{47}$ D. A. Sanders, ${ }^{47}$ D. J. Summers, ${ }^{47}$ H. W. Zhao, ${ }^{47}$ S. Brunet, ${ }^{48}$ D. Côté, ${ }^{48}$ P. Taras,${ }^{48}$ B. Viaud ${ }^{48}$ H. Nicholson, ${ }^{49}$ N. Cavallo, ${ }^{50,}$ G. De Nardo,${ }^{50}$ F. Fabozzi, ${ }^{50, \dagger}$ C. Gatto, ${ }^{50}$ L. Lista, ${ }^{50}$ D. Monorchio ${ }^{50}$ P. Paolucci, ${ }^{50}$ D. Piccolo,${ }^{50}$ C. Sciacca,${ }^{50}$ M. Baak,${ }^{51}$ H. Bulten, ${ }^{51}$ G. Raven, ${ }^{51}$ H. L. Snoek,${ }^{51}$ L. Wilden,${ }^{51}$ C. P. Jessop, ${ }^{52}$ J. M. LoSecco, ${ }^{52}$ T. Allmendinger, ${ }^{53}$ G. Benelli, ${ }^{53}$ K. K. Gan,${ }^{53}$ K. Honscheid, ${ }^{53}$ D. Hufnagel, ${ }^{53}$ 
P. D. Jackson, ${ }^{53}$ H. Kagan, ${ }^{53}$ R. Kass,${ }^{53}$ T. Pulliam, ${ }^{53}$ A. M. Rahimi, ${ }^{53}$ R. Ter-Antonyan, ${ }^{53}$ Q. K. Wong, ${ }^{53}$ J. Brau, ${ }^{54}$ R. Frey, ${ }^{54}$ O. Igonkina,${ }^{54}$ M. Lu ${ }^{54}$ C. T. Potter, ${ }^{54}$ N. B. Sinev, ${ }^{54}$ D. Strom,${ }^{54}$ J. Strube,${ }^{54}$ E. Torrence, ${ }^{54}$ A. Dorigo,${ }^{55}$ F. Galeazzi, ${ }^{55}$ M. Margoni, ${ }^{55}$ M. Morandin, ${ }^{55}$ M. Posocco,${ }^{55}$ M. Rotondo,${ }^{55}$ F. Simonetto, ${ }^{55}$ R. Stroili, ${ }^{55}$ C. Voci, ${ }^{55}$ M. Benayoun, ${ }^{56} \mathrm{H}$. Briand, ${ }^{56} \mathrm{~J}$. Chauveau, ${ }^{56} \mathrm{P}$. David, ${ }^{56} \mathrm{~L}$. Del Buono,${ }^{56} \mathrm{Ch}$. de la Vaissière, ${ }^{56}$ O. Hamon,${ }^{56} \mathrm{M}$. J. J. John, ${ }^{56}$ Ph. Leruste, ${ }^{56}$ J. Malclès, ${ }^{56}$ J. Ocariz,${ }^{56}$ L. Roos,${ }^{56}$ G. Therin,${ }^{56}$ P. K. Behera ${ }^{57}$ L. Gladney, ${ }^{57}$ Q. H. Guo,${ }^{57}$ J. Panetta, ${ }^{57}$ M. Biasini,${ }^{58}$ R. Covarelli, ${ }^{58}$ S. Pacetti, ${ }^{58}$ M. Pioppi, ${ }^{58}$ C. Angelini,${ }^{59}$ G. Batignani, ${ }^{59}$ S. Bettarini,${ }^{59}$ F. Bucci, ${ }^{59}$ G. Calderini, ${ }^{59}$ M. Carpinelli, ${ }^{59}$ R. Cenci, ${ }^{59}$ F. Forti, ${ }^{59}$ M. A. Giorgi,${ }^{59}$ A. Lusiani, ${ }^{59}$ G. Marchiori, ${ }^{59}$ M. Morganti,${ }^{59}$ N. Neri, ${ }^{59}$ E. Paoloni, ${ }^{59}$ M. Rama, ${ }^{59}$ G. Rizzo, ${ }^{59}$ J. Walsh, ${ }^{59}$ M. Haire,${ }^{60}$ D. Judd, ${ }^{60}$ D. E. Wagoner, ${ }^{60}$ J. Biesiada, ${ }^{61}$ N. Danielson, ${ }^{61}$ P. Elmer, ${ }^{61}$ Y.P. Lau, ${ }^{61}$ C. Lu, ${ }^{61}$ J. Olsen, ${ }^{61}$ A. J. S. Smith, ${ }^{61}$ A. V. Telnov, ${ }^{61}$ F. Bellini, ${ }^{62}$ G. Cavoto, ${ }^{62}$ A. D’ Orazio, ${ }^{62}$ E. Di Marco, ${ }^{62}$ R. Faccini, ${ }^{62}$ F. Ferrarotto, ${ }^{62}$ F. Ferroni,${ }^{62}$ M. Gaspero, ${ }^{62}$ L. Li Gioi, ${ }^{62}$ M. A. Mazzoni, ${ }^{62}$ S. Morganti, ${ }^{62}$ G. Piredda, ${ }^{62}$ F. Polci, ${ }^{62}$ F. Safai Tehrani, ${ }^{62}$ C. Voena, ${ }^{62}$ H. Schröder, ${ }^{63}$ G. Wagner, ${ }^{63}$ R. Waldi, ${ }^{63}$ T. Adye,${ }^{64}$ N. De Groot,${ }^{64}$ B. Franek, ${ }^{64}$ G. P. Gopal,${ }^{64}$ E. O. Olaiya, ${ }^{64}$ F. F. Wilson, ${ }^{64}$ R. Aleksan, ${ }^{65}$ S. Emery, ${ }^{65}$ A. Gaidot, ${ }^{65}$

S. F. Ganzhur, ${ }^{65}$ P.-F. Giraud, ${ }^{65}$ G. Graziani, ${ }^{65}$ G. Hamel de Monchenault, ${ }^{65}$ W. Kozanecki, ${ }^{65}$ M. Legendre, ${ }^{65}$ G. W. London, ${ }^{65}$ B. Mayer, ${ }^{65}$ G. Vasseur, ${ }^{65}$ Ch. Yèche, ${ }^{65}$ M. Zito, ${ }^{65}$ M. V. Purohit ${ }^{66}$ A. W. Weidemann, ${ }^{66}$ J. R. Wilson, ${ }^{66}$ F. X. Yumiceva, ${ }^{66}$ T. Abe,${ }^{67}$ M. T. Allen,${ }^{67}$ D. Aston, ${ }^{67}$ R. Bartoldus, ${ }^{67}$ N. Berger, ${ }^{67}$ A. M. Boyarski, ${ }^{67}$ O. L. Buchmueller, ${ }^{67}$ R. Claus ${ }^{67}$ M. R. Convery, ${ }^{67}$ M. Cristinziani, ${ }^{67}$ J. C. Dingfelder, ${ }^{67}$ D. Dong,${ }^{67}$ J. Dorfan ${ }^{67}$ D. Dujmic, ${ }^{67}$ W. Dunwoodie,${ }^{67}$ S. Fan, ${ }^{67}$ R. C. Field, ${ }^{67}$ T. Glanzman,${ }^{67}$ S. J. Gowdy, ${ }^{67}$ T. Hadig, ${ }^{67}$ V. Halyo, ${ }^{67}$ C. Hast, ${ }^{67}$ T. Hryn'ova,${ }^{67}$ W. R. Innes, ${ }^{67}$ M. H. Kelsey, ${ }^{67}$ P. Kim, ${ }^{67}$ M. L. Kocian, ${ }^{67}$ D. W. G. S. Leith, ${ }^{67}$ J. Libby, ${ }^{67}$ S. Luitz, ${ }^{67}$ V. Luth, ${ }^{67}$ H. L. Lynch, ${ }^{67}$ H. Marsiske, ${ }^{67}$ R. Messner, ${ }^{67}$ D. R. Muller, ${ }^{67}$ C. P. O'Grady, ${ }^{67}$ V. E. Ozcan, ${ }^{67}$ A. Perazzo, ${ }^{67}$ M. Perl, ${ }^{67}$ B. N. Ratcliff,,${ }^{67}$ A. Roodman, ${ }^{67}$ A. A. Salnikov, ${ }^{67}$ R. H. Schindler, ${ }^{67}$ J. Schwiening, ${ }^{67}$ A. Snyder,${ }^{67}$ J. Stelzer, ${ }^{67}$ D. Su, ${ }^{67}$ M. K. Sullivan, ${ }^{67}$ K. Suzuki, ${ }^{67}$ S. Swain, ${ }^{67}$ J. M. Thompson, ${ }^{67}$ J. Va'vra, ${ }^{67}$ M. Weaver, ${ }^{67}$ W. J. Wisniewski, ${ }^{67}$ M. Wittgen, ${ }^{67}$ D. H. Wright,${ }^{67}$ A. K. Yarritu, ${ }^{67}$ K. Yi, ${ }^{67}$ C. C. Young, ${ }^{67}$ P. R. Burchat, ${ }^{68}$ A. J. Edwards, ${ }^{68}$ S. A. Majewski, ${ }^{68}$ B. A. Petersen, ${ }^{68}$ C. Roat, ${ }^{68}$ M. Ahmed, ${ }^{69}$ S. Ahmed, ${ }^{69}$ M. S. Alam, ${ }^{69}$ J. A. Ernst ${ }^{69}$ M. A. Saeed, ${ }^{69}$ F. R. Wappler, ${ }^{69}$ S. B. Zain, ${ }^{69}$ W. Bugg, ${ }^{70}$ M. Krishnamurthy, ${ }^{70}$ S. M. Spanier, ${ }^{70}$ R. Eckmann, ${ }^{71}$ J. L. Ritchie, ${ }^{71}$ A. Satpathy, ${ }^{71}$ R. F. Schwitters, ${ }^{71}$ J. M. Izen, ${ }^{72}$ I. Kitayama, ${ }^{72}$ X. C. Lou, ${ }^{72}$ S. Ye,${ }^{72}$ F. Bianchi, ${ }^{73}$ M. Bona ${ }^{73}$ F. Gallo, ${ }^{73}$ D. Gamba ${ }^{73}$ M. Bomben,${ }^{74}$ L. Bosisio, ${ }^{74}$ C. Cartaro ${ }^{74}$ F. Cossutti, ${ }^{74}$ G. Della Ricca, ${ }^{74}$ S. Dittongo,${ }^{74}$ S. Grancagnolo,${ }^{74}$ L. Lanceri, ${ }^{74}$ L. Vitale,${ }^{74}$

F. Martinez-Vidal,${ }^{75}$ R. S. Panvini, ${ }^{76, \$}$ Sw. Banerjee, ${ }^{77}$ B. Bhuyan, ${ }^{77}$ C. M. Brown,${ }^{77}$ D. Fortin, ${ }^{77}$ K. Hamano,${ }^{77}$ R. Kowalewski, ${ }^{77}$ J. M. Roney, ${ }^{77}$ R. J. Sobie, ${ }^{77}$ J. J. Back, ${ }^{78}$ P. F. Harrison, ${ }^{78}$ T. E. Latham, ${ }^{78}$ G. B. Mohanty, ${ }^{78}$ H. R. Band,${ }^{79}$ X. Chen, ${ }^{79}$ B. Cheng, ${ }^{79}$ S. Dasu, ${ }^{79}$ M. Datta, ${ }^{79}$ A. M. Eichenbaum, ${ }^{79}$ K. T. Flood ${ }^{79}$ M. Graham, ${ }^{79}$ J. J. Hollar, ${ }^{79}$ J. R. Johnson, ${ }^{79}$ P.E. Kutter, ${ }^{79}$ H. Li,${ }^{79}$ R. Liu, ${ }^{79}$ B. Mellado, ${ }^{79}$ A. Mihalyi ${ }^{79}$ Y. Pan, ${ }^{79}$ R. Prepost, ${ }^{79}$ P. Tan, ${ }^{79}$ J. H. von Wimmersperg-Toeller, ${ }^{79}$ S. L. Wu ${ }^{79}$ Z. Yu, ${ }^{79}$ and H. Neal ${ }^{80}$

(BABAR Collaboration)

${ }^{1}$ Laboratoire de Physique des Particules, F-74941 Annecy-le-Vieux, France

${ }^{2}$ IFAE, Universitat Autonoma de Barcelona, E-08193 Bellaterra, Barcelona, Spain

${ }^{3}$ Università di Bari, Dipartimento di Fisica and INFN, I-70126 Bari, Italy

${ }^{4}$ Institute of High Energy Physics, Beijing 100039, China

${ }^{5}$ Institute of Physics, University of Bergen, N-5007 Bergen, Norway

${ }^{6}$ Lawrence Berkeley National Laboratory, Berkeley, California 94720, USA and University of California, Berkeley, California 94720, USA

${ }^{7}$ University of Birmingham, Birmingham, B15 2TT, United Kingdom

${ }^{8}$ Institut für Experimentalphysik 1, Ruhr Universität Bochum, D-44780 Bochum, Germany

${ }^{9}$ University of Bristol, Bristol BS8 1TL, United Kingdom

${ }^{10}$ University of British Columbia, Vancouver, British Columbia V6T 1Z1, Canada

${ }^{11}$ Brunel University, Uxbridge, Middlesex UB8 3PH, United Kingdom

${ }^{12}$ Budker Institute of Nuclear Physics, Novosibirsk 630090, Russia

${ }^{13}$ University of California at Irvine, Irvine, California 92697, USA

${ }^{14}$ University of California at Los Angeles, Los Angeles, California 90024, USA

${ }^{15}$ University of California at Riverside, Riverside, California 92521, USA

${ }^{16}$ University of California at San Diego, La Jolla, California 92093, USA

${ }^{17}$ University of California at Santa Barbara, Santa Barbara, California 93106, USA

${ }^{18}$ Institute for Particle Physics, University of California at Santa Cruz, Santa Cruz, California 95064, USA

${ }^{19}$ California Institute of Technology, Pasadena, California 91125, USA 


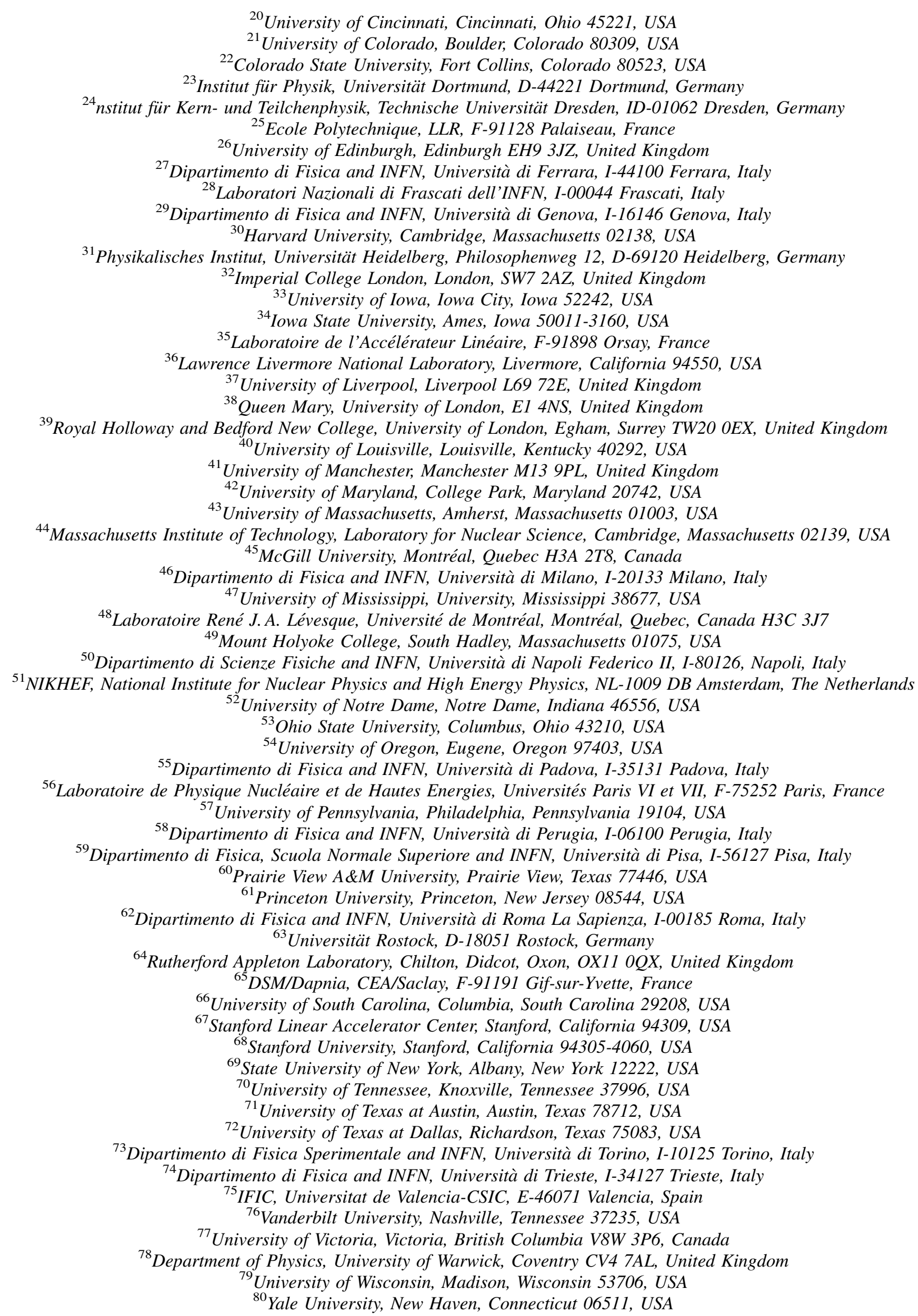


(Received 23 June 2005; published 1 November 2005)

A search for lepton-flavor and lepton-number violation in the decay of the tau lepton into one charged lepton and two charged hadrons is performed using $221.4 \mathrm{fb}^{-1}$ of data collected at an $e^{+} e^{-}$center-ofmass energy of $10.58 \mathrm{GeV}$ with the BABAR detector at the SLAC PEP-II storage ring. In all 14 decay modes considered, the observed data are compatible with background expectations, and upper limits are set in the range $\mathcal{B}\left(\tau \rightarrow \ell h h^{\prime}\right)<(0.7-4.8) \times 10^{-7}$ at $90 \%$ confidence level.

DOI: $10.1103 /$ PhysRevLett.95.191801

PACS numbers: 13.35.Dx, 11.30.Fs, 11.30.Hv, 14.60.Fg

Lepton-flavor violation (LFV) involving charged leptons has never been observed, and there are stringent experimental limits from muon decays: $\mathcal{B}(\mu \rightarrow$ $e \gamma)<1.2 \times 10^{-11}[1]$ and $\mathcal{B}(\mu \rightarrow e e e)<1.0 \times 10^{-12}$ [2] at $90 \%$ confidence level (C.L.). In tau decays, the most stringent limits on LFV are $\mathcal{B}(\tau \rightarrow \mu \gamma)<6.8 \times$ $10^{-8}$ and $\mathcal{B}(\tau \rightarrow \ell \ell \ell)<(1-3) \times 10^{-7}$ at $90 \%$ C.L. $[3,4]$. While forbidden in the standard model (SM), many extensions to the SM predict enhanced LFV in tau decays with respect to muon decays with branching fractions from $10^{-10}$ up to the current experimental limits [5]. Observation of LFV in tau decays would be a clear signature of physics beyond the SM, while nonobservation will provide further constraints on theoretical models.

This Letter presents the results of a search for leptonflavor violation in the neutrinoless decays $\tau^{-} \rightarrow \ell^{-} h^{+} h^{-}$ where $\ell$ represents an electron or muon and $h$ represents a pion or kaon [6]. In addition, a search is also performed for the decays $\tau^{-} \rightarrow \ell^{+} h^{-} h^{-}$which also violate leptonnumber conservation. All possible lepton and hadron combinations consistent with charge conservation are considered, leading to 14 distinct decay modes as shown in Table I. The best existing limits on the branching fractions for these decay modes currently come from CLEO: $(2-8) \times 10^{-6}$ at $90 \%$ C.L. [7].

The data used in this analysis were collected with the $B A B A R$ detector at the SLAC PEP-II asymmetric-energy $e^{+} e^{-}$storage ring. The data sample consists of $221.4 \mathrm{fb}^{-1}$ recorded at a luminosity-weighted center-of-mass energy $\sqrt{s}=10.58 \mathrm{GeV}$. With an estimated cross section for tau pairs of $\sigma_{\tau \tau}=(0.89 \pm 0.02) \mathrm{nb}$ [8], this data sample contains nearly $4 \times 10^{8}$ tau decays.

Charged-particle (track) momenta are measured with a 5-layer double-sided silicon vertex tracker and a 40-layer drift chamber inside a $1.5 \mathrm{~T}$ superconducting solenoidal magnet. An electromagnetic calorimeter (EMC) consisting of $6580 \mathrm{CsI}(\mathrm{Tl})$ crystals is used to identify electrons and photons, a ring-imaging Cherenkov detector (DIRC) and energy loss in the tracking system are used to identify charged hadrons, and the instrumented magnetic flux return (IFR) is used to identify muons. Further details on the $B A B A R$ detector are found in Ref. [9].

A Monte Carlo (MC) simulation of neutrinoless tau decays is used to study the performance of this analysis. Simulated $\tau^{+} \tau^{-}$events including higher-order radiative corrections are generated using the $\mathrm{KK} 2 \mathrm{~F}$ MC generator [8], with one tau decaying to one lepton and two hadrons with a 3-body phase space distribution, while the second tau decay is simulated with TAUOLA [10] according to measured rates [11]. Final state radiative effects are simulated for all decays using РнотоS [12]. The detector response is simulated with GEANT [13], and the simulated events are reconstructed in the same manner as data.

Candidate signal events are required to have a 1-3 topology, where one tau decay yields one charged particle (1 prong), while the other tau decay yields three charged particles (3 prong). Four well reconstructed tracks are required with zero net charge, originating from a common region consistent with $\tau \tau$ production and decay. Pairs of oppositely charged tracks, likely to be from photon conversions in the detector material, are ignored if their $e^{+} e^{-}$ invariant mass is less than $30 \mathrm{MeV} / c^{2}$. The event is divided into hemispheres using the plane perpendicular to the thrust axis, calculated from the observed track momenta and EMC energy deposits, in the center-of-mass (c.m.) frame. One hemisphere must contain exactly one track while the other must contain exactly three.

TABLE I. Efficiency estimates, the number of expected background events $\left(N_{\mathrm{bgd}}\right)$ in the signal region (with total uncertainties), the number of observed events $\left(N_{\mathrm{obs}}\right)$ in the signal region, and the $90 \%$ C.L. upper limit on the branching fraction for each decay mode.

\begin{tabular}{lcccc}
\hline \hline Mode & Efficiency [\%] & $N_{\text {bgd }}$ & $N_{\text {obs }}$ & UL at 90\% C.L. \\
\hline$e^{-} K^{+} K^{-}$ & $3.77 \pm 0.16$ & $0.22 \pm 0.06$ & 0 & $1.4 \times 10^{-7}$ \\
$e^{-} K^{+} \pi^{-}$ & $3.08 \pm 0.13$ & $0.32 \pm 0.08$ & 0 & $1.7 \times 10^{-7}$ \\
$e^{-} \pi^{+} K^{-}$ & $3.10 \pm 0.13$ & $0.14 \pm 0.06$ & 1 & $3.2 \times 10^{-7}$ \\
$e^{-} \pi^{+} \pi^{-}$ & $3.30 \pm 0.15$ & $0.81 \pm 0.13$ & 0 & $1.2 \times 10^{-7}$ \\
$\mu^{-} K^{+} K^{-}$ & $2.16 \pm 0.12$ & $0.24 \pm 0.07$ & 0 & $2.5 \times 10^{-7}$ \\
$\mu^{-} K^{+} \pi^{-}$ & $2.97 \pm 0.16$ & $1.67 \pm 0.29$ & 2 & $3.2 \times 10^{-7}$ \\
$\mu^{-} \pi^{+} K^{-}$ & $2.87 \pm 0.16$ & $1.04 \pm 0.18$ & 1 & $2.6 \times 10^{-7}$ \\
$\mu^{-} \pi^{+} \pi^{-}$ & $3.40 \pm 0.19$ & $2.99 \pm 0.41$ & 3 & $2.9 \times 10^{-7}$ \\
$e^{+} K^{-} K^{-}$ & $3.85 \pm 0.16$ & $0.04 \pm 0.04$ & 0 & $1.5 \times 10^{-7}$ \\
$e^{+} K^{-} \pi^{-}$ & $3.19 \pm 0.14$ & $0.16 \pm 0.06$ & 0 & $1.8 \times 10^{-7}$ \\
$e^{+} \pi^{-} \pi^{-}$ & $3.40 \pm 0.15$ & $0.41 \pm 0.10$ & 1 & $2.7 \times 10^{-7}$ \\
$\mu^{+} K^{-} K^{-}$ & $2.06 \pm 0.11$ & $0.07 \pm 0.10$ & 1 & $4.8 \times 10^{-7}$ \\
$\mu^{+} K^{-} \pi^{-}$ & $2.85 \pm 0.16$ & $1.54 \pm 0.25$ & 1 & $2.2 \times 10^{-7}$ \\
$\mu^{+} \pi^{-} \pi^{-}$ & $3.30 \pm 0.18$ & $1.46 \pm 0.27$ & 0 & $0.7 \times 10^{-7}$ \\
\hline \hline
\end{tabular}


One of the charged particles found in the 3-prong hemisphere must be identified as either an electron or muon candidate. Electrons are identified using the ratio of observed EMC energy to track momentum $(E / p)$, the shape of the shower in the EMC, and the ionization loss in the tracking system $(d E / d x)$. Muons are identified by hits in the IFR and small energy deposits in the EMC. Each of the other two charged particles found in the 3-prong hemisphere must be identified as either a pion or a kaon, using information from the DIRC and $d E / d x$.

After event topology and particle identification requirements, there are significant backgrounds from light quark $q \bar{q}$ production and SM $\tau \tau$ events (without LFV), as well as small contributions from Bhabha, $\mu^{+} \mu^{-}$, and two-photon production of four charged particles. Additional selection criteria, largely the same for all 14 signal channels, are applied as follows. No photon candidates, identified as EMC energy deposits unassociated to a track, with $E_{\gamma}>$ $100 \mathrm{MeV}$ are allowed. This restriction removes $q \bar{q}$ backgrounds and SM $\tau \tau$ events. The total transverse momentum of the event in the c.m. frame must be greater than $0.2 \mathrm{GeV} / c$, while the polar angle of the missing momentum in the laboratory frame is required to be in the range $[0.25,2.4]$ radians. These two requirements are effective at reducing two-photon and Bhabha backgrounds. The mass of the 1-prong hemisphere calculated from the fourmomentum of the track in the 1-prong hemisphere and the missing momentum in the event, is required to be in the range $[0.6,1.9] \mathrm{GeV} / c^{2}$ for $e h h^{\prime}$ candidates and $[0.8,1.9] \mathrm{GeV} / c^{2}$ for $\mu h h^{\prime}$ candidates. The 1-prong mass requirement is particularly effective at removing $q \bar{q}$ backgrounds as well as the remaining two-photon contribution. To reduce Bhabha backgrounds, the momentum of the 1prong track in the c.m. frame is required to be less than $4.5 \mathrm{GeV} / c$ for the $e \pi \pi$ candidates. In addition, particle identification vetoes are applied to specific selection channels. For all decay modes, lepton and pion candidates must not pass the kaon identification as well. For the $e h h^{\prime}$ decay modes, except for $e K K$, the 1-prong track must not be identified as an electron. This requirement is useful to reduce possible contamination from Bhabhas.

To further reduce backgrounds, candidate signal events are required to have an invariant mass and total energy in the 3-prong hemisphere consistent with the neutrinoless decay of a tau lepton. These quantities are calculated from the observed track momenta assuming the corresponding lepton and hadron masses for each decay mode. The mass difference and energy difference are defined as $\Delta M \equiv M_{\mathrm{rec}}-m_{\tau}$ and $\Delta E \equiv E_{\mathrm{rec}}^{\text {c.m. }}-E_{\text {beam }}^{\text {c.m. }}$, where $M_{\text {rec }}$ is the reconstructed 3-prong invariant mass, $m_{\tau}=$ $1.777 \mathrm{GeV} / c^{2}$ is the tau mass [14], $E_{\text {rec }}^{\text {c.m. }}$ is the reconstructed 3-prong total energy in the c.m. frame, and $E_{\text {beam }}^{\text {c.m. }}$ is the c.m. beam energy. Rectangular signal regions are defined separately for each decay mode in the $(\Delta M, \Delta E)$ plane. For the $\mu h h^{\prime}$ modes, $\Delta M$ is required to be in the range $[-20,+20] \mathrm{MeV} / c^{2}$, while for the $e h h^{\prime}$ modes the range is $[-30,+20] \mathrm{MeV} / c^{2}$ to account for radiative losses. For all 14 decay modes, $\Delta E$ must be in the range $[-100,+50] \mathrm{MeV}$.

These signal region boundaries are optimized to provide the smallest expected upper limits on the branching fractions in the background-only hypothesis. These expected upper limits are estimated using only MC simulations, not candidate events in data. To avoid bias, a blind analysis procedure was adopted with the number of data events in the signal region remaining unknown until the selection criteria were finalized and all systematic studies had been performed. Figure 1 shows the observed data for all 14 selection channels, along with the signal region boundaries and the expected signal distributions.

The dominant remaining backgrounds are low multiplicity $q \bar{q}$ events and SM $\tau \tau$ events. These background classes have unique distributions in the $(\Delta M, \Delta E)$ plane: $q \bar{q}$ events populate the plane uniformly, while $\tau \tau$ backgrounds are restricted to negative values of both $\Delta M$ and $\Delta E$. Backgrounds from Bhabha, $\mu \mu$, and two-photon

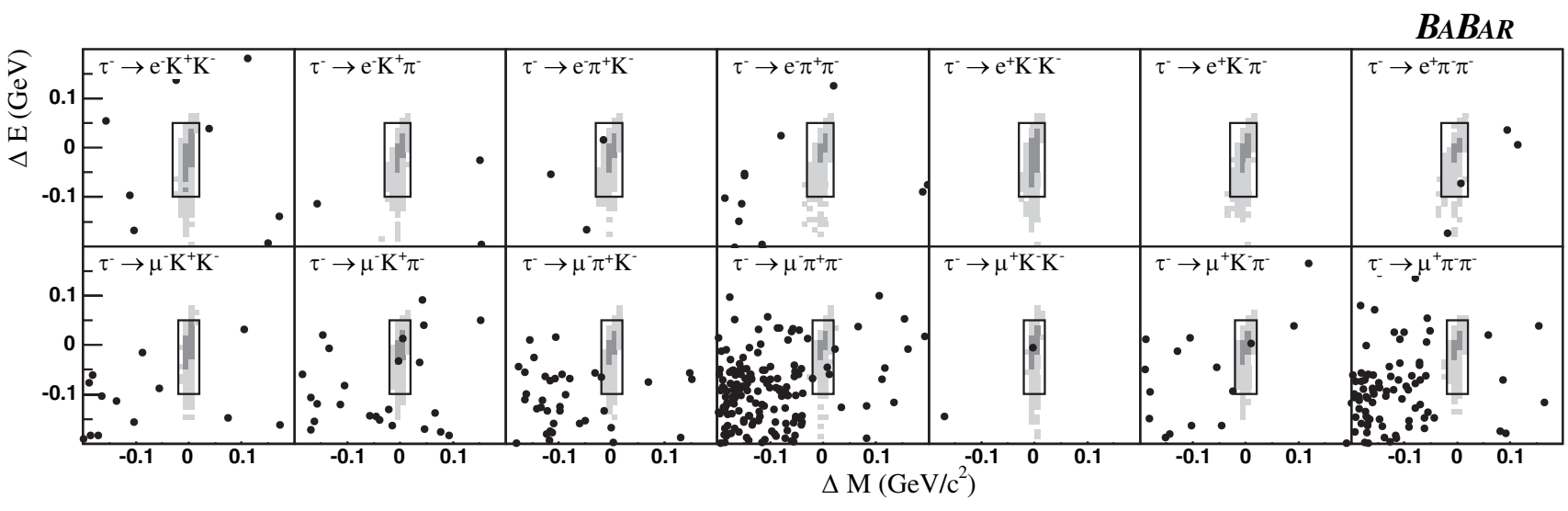

FIG. 1. Observed data shown as dots in the $(\Delta M, \Delta E)$ plane and the boundaries of the signal region for each decay mode. The dark and light shading indicates contours containing $50 \%$ and $90 \%$ of the selected MC signal events, respectively. 
events are found to be negligible. For each background class, a probability density function (PDF) describing the shape of the background distribution in the $(\Delta M, \Delta E)$ plane is determined by fitting an analytic function to the Monte Carlo prediction as described in more detail below. These PDFs are then combined with normalization coefficients determined from an unbinned maximum likelihood fit to the observed data in the $(\Delta M, \Delta E)$ plane in a sideband $(\mathrm{SB})$ region. The resulting function describes the event rate observed in the SB region and is used to predict the expected background rate in the signal region. The SB region is defined as the rectangle, excluding the signal region, bounding $\Delta M$ in the range $[-0.7,+0.4] \mathrm{GeV} / c^{2}$ for $e h h^{\prime}$ final states and $[-0.4,+0.4] \mathrm{GeV} / c^{2}$ for $\mu h h^{\prime}$ final states, while $\Delta E$ must be in the range $[-0.7,+0.4] \mathrm{GeV}$. The PDF shape determinations and SB fits are performed separately for each of the 14 decay modes.

For the $q \bar{q}$ backgrounds, a PDF is constructed from the product of two functions $P_{M^{\prime}}$ and $P_{E^{\prime}}$, where the coordinates $\left(\Delta M^{\prime}, \Delta E^{\prime}\right)$ have been rotated slightly from $(\Delta M, \Delta E)$ to better fit the expected distributions. The function $P_{M^{\prime}}\left(\Delta M^{\prime}\right)$ is a Gaussian and the function $P_{E^{\prime}}\left(\Delta E^{\prime}\right)=\left(1-x / \sqrt{1+x^{2}}\right)\left(1+a_{1} x+a_{2} x^{2}+a_{3} x^{3}\right)$ where $x=\left(\Delta E^{\prime}-a_{4}\right) / a_{5}$ and $a_{i}$ are fit parameters. The resulting $q \bar{q}$ PDF is described by eight fit parameters, including the rotation angle, which are determined by fits to MC $q \bar{q}$ background samples for each decay mode. For the $\tau \tau$ PDF, the function $P_{M^{\prime}}\left(\Delta M^{\prime}\right)$ is the sum of two Gaussians with different widths above and below the peak, while the functional form of $P_{E^{\prime}}\left(\Delta E^{\prime}\right)$ is the same as the $q \bar{q}$ PDF above. To properly model the wedge-shaped kinematic limit in tau decays, a coordinate transformation of the form $\Delta M^{\prime}=\cos \beta_{1} \Delta M+\sin \beta_{1} \Delta E$ and $\Delta E^{\prime}=$ $\cos \beta_{2} \Delta E-\sin \beta_{2} \Delta M$ is performed. In total there are 12 free parameters describing this PDF, and all are determined by fits to MC $\tau \tau$ samples.

With the shapes of the two background PDFs determined, an unbinned maximum likelihood fit to the data in the SB region is used to find the expected rate of each background type in the signal region. Extensive MC studies show that these PDF functions adequately describe the predicted background shapes near the signal regions. The accuracy of these predictions is verified by comparing to data in regions neighboring the signal region in the $(\Delta M, \Delta E)$ plane where no signal is expected. Expected backgrounds are shown in Table I, and an example of the background prediction compared to the observed data is shown in Fig. 2.

The efficiency of the selection for signal events is estimated with a MC simulation of neutrinoless tau decays. About $40 \%$ of the MC signal events pass the initial 1-3 topology requirement, and $20 \%$ to $70 \%$ of these preselected events pass the particle identification (PID) criteria, depending upon the signal mode. The final efficiency for signal events to be found in the signal region after all

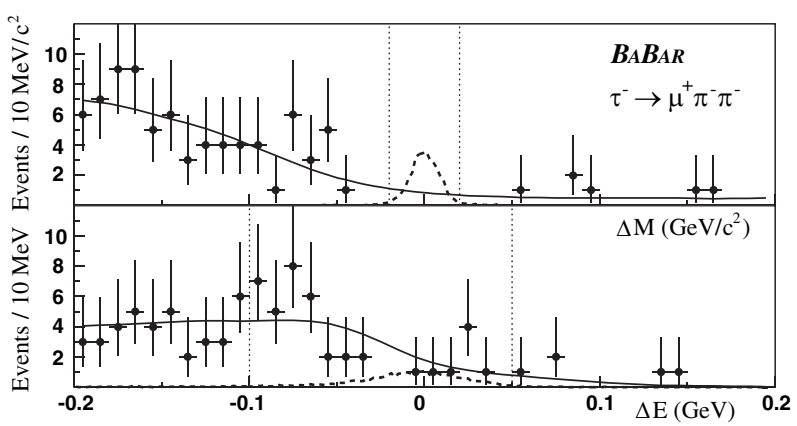

FIG. 2. Data (points) and background expectation (solid line) are shown for the $\mu^{+} \pi^{-} \pi^{-}$candidates displayed in Fig. 1. Expected signal distributions for a branching fraction of $5 \times$ $10^{-7}$ are also shown as the dashed curve. The vertical lines indicate the signal region.

requirements is shown in Table I for each decay mode and ranges from $2.1 \%$ to $3.8 \%$. This efficiency includes the $85 \%$ branching fraction for 1-prong tau decays [11].

The PID selection efficiencies and misidentification rates are measured directly using tracks in kinematicallyselected data control samples. These values are parametrized as a function of particle momentum, charge, polar angle, and azimuthal angle in the laboratory frame. The lepton-identification criteria have been designed to give very low misidentification rates at the expense of some efficiency loss. The electron ID is expected to be $81 \%$ efficient in signal $e h h^{\prime}$ events, with a mis-ID rate of $0.1 \%$ for pions and $0.2 \%$ for kaons in generic $\tau \tau$ events. The muon ID is $44 \%$ efficient for $\mu h h^{\prime}$ signal events, with a mis-ID rate of $1.0 \%$ for pions and $0.4 \%$ for kaons. The hadronic identification is designed to classify the hadronic candidates as pions or kaons, but is not intended to distinguish hadrons from leptons. The pion ID is $92 \%$ efficient with a mis-ID rate of $12 \%$ for kaons, while the kaon ID is $81 \%$ efficient with a $1.4 \%$ mis-ID rate for pions.

The largest systematic uncertainty for the signal efficiency is the uncertainty in measuring particle ID efficiencies. This uncertainty (all uncertainties quoted are relative) is dominated by the statistical precision of the PID control samples, and ranges from $0.7 \%$ for $e^{-} \pi^{+} \pi^{-}$to $3.8 \%$ for $\mu^{-} K^{+} K^{-}$. The modeling of the tracking efficiency contributes an uncertainty of $2.5 \%$, while the restriction on extra photons leads to an additional uncertainty of $2.4 \%$. All other sources of uncertainty are found to be small, including the modeling of radiative effects, track momentum resolution, trigger performance, observables used in the selection criteria, and knowledge of the tau 1-prong branching fractions. No uncertainty is assigned for possible model dependence of the signal decay. The selection efficiency is found to be uniform within $20 \%$ across the Dalitz plane, provided the invariant mass for any pair of particles is less than $1.4 \mathrm{GeV} / c^{2}$.

Since the background levels are extracted directly from the data, systematic uncertainties on the background esti- 
mation are directly related to the background normalization, parametrization, and the fit technique used. The finite data available in the SB region used to determine the background rates dominates the background uncertainty. Additional uncertainties of $10 \%$ are estimated by varying the fit procedure and changing the functional form of the background PDFs. The uncertainty on the branching fraction of SM tau decays with one or two kaons is also evaluated, and contributes less than $15 \%$ for all final states.

The numbers of events observed $\left(N_{\text {obs }}\right)$ and the background expectations $\left(N_{\text {bgd }}\right)$ are shown in Table I, with no significant excess observed. Upper limits on the branching fractions are calculated according to $\mathcal{B}_{\mathrm{UL}}^{90}=N_{\mathrm{UL}}^{90} /$ $\left(2 \varepsilon \mathcal{L} \sigma_{\tau \tau}\right)$, where $N_{\mathrm{UL}}^{90}$ is the $90 \%$ C.L. upper limit for the number of signal events when $N_{\text {obs }}$ events are observed with $N_{\text {bgd }}$ background events expected. The quantities $\varepsilon$, $\mathcal{L}$, and $\sigma_{\tau \tau}$ are the selection efficiency, luminosity, and $\tau^{+} \tau^{-}$cross section, respectively. The branching fraction upper limits are calculated including all uncertainties using the technique of Cousins and Highland [15] following the implementation of Barlow [16]. The estimates of $\mathcal{L}$ and $\sigma_{\tau \tau}$ are correlated [17], and the uncertainty on the product $\mathcal{L} \sigma_{\tau \tau}$ is $2.3 \%$. The $90 \%$ C.L. upper limits on the $\tau \rightarrow \ell h h^{\prime}$ branching fractions, shown in Table I, are in the range $(0.7-4.8) \times 10^{-7}$. These limits represent an order of magnitude improvement over the previous experimental bounds [7].

We are grateful for the excellent luminosity and machine conditions provided by our SLAC PEP-II colleagues, and for the substantial dedicated effort from the computing organizations that support BABAR. The collaborating institutions wish to thank SLAC for its support and kind hospitality. This work is supported by DOE and NSF (USA), NSERC (Canada), IHEP (China), CEA and CNRS-IN2P3 (France), BMBF and DFG (Germany), INFN (Italy), FOM (The Netherlands), NFR (Norway), MIST (Russia), and PPARC (United Kingdom). Individuals have received support from CONACyT
(Mexico), A.P. Sloan Foundation, Research Corporation, and Alexander von Humboldt Foundation.

*Also with Università di Perugia, Dipartimento di Fisica, Perugia, Italy.

${ }^{\dagger}$ Also with Università della Basilicata, Potenza, Italy. "Deceased.

[1] M. L. Brooks et al. (MEGA/LAMPF Collaboration), Phys. Rev. Lett. 83, 1521 (1999).

[2] U. Bellgardt et al. (SINDRUM Collaboration), Nucl. Phys. B299, 1 (1988).

[3] B. Aubert et al. (BABAR Collaboration), Phys. Rev. Lett. 95, 041802 (2005).

[4] B. Aubert et al. (BABAR Collaboration), Phys. Rev. Lett. 92, 121801 (2004).

[5] E. Ma, Nucl. Phys. B, Proc. Suppl. 123, 125 (2003).

[6] Throughout this Letter, charge conjugate decay modes are also implied.

[7] D. W. Bliss et al. (CLEO Collaboration), Phys. Rev. D 57, 5903 (1998).

[8] B. F. Ward, S. Jadach, and Z. Was, Nucl. Phys. B, Proc. Suppl. 116, 73 (2003).

[9] B. Aubert et al. (BABAR Collaboration), Nucl. Instrum. Methods Phys. Res., Sect. A 479, 1 (2002).

[10] S. Jadach, Z. Was, R. Decker, and J. H. Kuhn, Comput. Phys. Commun. 76, 361 (1993).

[11] S. Eidelman et al. (Particle Data Group), Phys. Lett. B 592, 1 (2004).

[12] E. Barberio and Z. Was, Comput. Phys. Commun. 79, 291 (1994).

[13] S. Agostinelli et al. (GEANT4 Collaboration), Nucl. Instrum. Methods Phys. Res., Sect. A 506, 250 (2003).

[14] J.Z. Bai et al. (BES Collaboration), Phys. Rev. D 53, 20 (1996).

[15] R. D. Cousins and V. L. Highland, Nucl. Instrum. Methods Phys. Res., Sect. A 320, 331 (1992).

[16] R. Barlow, Comput. Phys. Commun. 149, 97 (2002).

[17] The luminosity is measured using the observed $\mu^{+} \mu^{-}$ rate, and the $\mu^{+} \mu^{-}$and $\tau^{+} \tau^{-}$cross sections are both estimated with KK2F. 